\title{
Atomic-number dependence of the magnetic-sublevel population in the autoionization state formed in dielectronic recombination
}

\author{
Zhimin Hu, $, 1,2,3,{ }^{*}$ Yueming Li, ${ }^{4}$ Xiaoying Han, ${ }^{4}$ Daiji Kato, ${ }^{5,6}$ Xiaomin Tong,${ }^{7,8}$ Hirofumi Watanabe, ${ }^{9}$ and Nobuyuki Nakamura ${ }^{1}$ \\ ${ }^{1}$ Institute for Laser Science, The University of Electro-Communications, Chofu, Tokyo 182-8585, Japan \\ ${ }^{2}$ Research Center of Laser Fusion, China Academy of Engineering Physics, Mianyang 621900, China \\ ${ }^{3}$ Physics Institute, Heidelberg University, Im Neuenheimer Feld 226, 69120 Heidelberg, Germany \\ ${ }^{4}$ Institute of Applied Physics and Computational Mathematics, P. O. Box 8009, Beijing 100088, China \\ ${ }^{5}$ National Institute for Fusion Science, Toki, Gifu 509-5292, Japan \\ ${ }^{6}$ Department of Fusion Science, The Graduate University of Advanced Studies (SOKENDAI), Toki, Gifu 509-5292, Japan \\ ${ }^{7}$ Division of Materials Science, Faculty of Pure and Applied Science, University of Tsukuba, Tsukuba, Ibaraki 305-8573, Japan \\ ${ }^{8}$ Center for Computational Sciences, University of Tsukuba, Tsukuba, Ibaraki 305-8573, Japan \\ ${ }^{9}$ Center of Applied Superconductivity and Sustainable Energy Research, Chubu University, Kasugai, Aichi 487-8501, Japan
}

(Received 28 August 2014; revised manuscript received 15 October 2014; published 1 December 2014)

\begin{abstract}
The magnetic-sublevel population of the autoionization state formed in dielectronic recombination (DR) of highly charged heavy ions has been experimentally investigated by combining two types of measurements with an electron beam ion trap. The two different measurements are the differential x-ray measurement at $90^{\circ}$ with respect to the electron beam and the integral resonance-strength measurement. The alignment parameter, which denotes the magnetic-sublevel population distribution, has been obtained for the autoionization states $\left[1 s 2 s^{2} 2 p_{1 / 2}\right]_{1}$ formed in DR of Li-like $\operatorname{Pr}$ (atomic number $\left.Z=59\right)$ and Ho $(Z=67)$. This work is a complement to our previous work [Phys. Rev. Lett. 108, 073002 (2012)] on Li-like Au $(Z=79)$. The experimental results are in reasonable agreement with theoretical predictions, and a strong $Z$-dependent tendency due to the Breit interaction has been confirmed.
\end{abstract}

DOI: 10.1103/PhysRevA.90.062702

PACS number(s): 34.80.Lx, 32.30.Rj

\section{INTRODUCTION}

Photon emission from atoms or ions excited by a unidirectional electron beam generally has an anisotropic angular distribution or polarization characteristics, due to the uneven population of the magnetic sublevels. The degrees of anisotropy and polarization depend on the extent of the deviation from an even population of the excited magnetic sublevels [1]. The anisotropy and polarization can thus be used as a diagnostic tool for the nonthermal component in plasmas [2-5].

Dielectronic recombination (DR) of highly charged ions is one of the most important $\mathrm{X}$-ray emission processes in hot plasmas. DR is the combination of dielectronic capture (DC) and successive radiative stablization (RS):

$$
e^{-}+A^{q+} \stackrel{\mathrm{DC}}{\rightarrow} A^{(q-1)+* *} \stackrel{\mathrm{RS}}{\rightarrow} A^{(q-1)+}+h \nu .
$$

Since it is a resonant process having a large $\mathrm{x}$-ray emission cross section at the resonance energy, the anisotropy of $x$ rays emitted in DR is important for hot-plasma diagnostics [6,7]. The anisotropy and polarization of x-ray emissions in the DR process have thus been investigated so far extensively in theory [8-12], but rarely in experiments due to technical difficulties especially for few-electron heavy ions.

There are mainly two experimental devices that are suited for studying collision processes of few-electron heavy ions; one is an electron-beam ion trap (EBIT) $[13,14]$ and the other is an ion storage ring [15,16]. Shlyaptseva et al. [17] studied the polarization of the $\mathrm{x}$ rays emitted in DR of Li-like Fe using an EBIT. In their study, the polarization

\footnotetext{
*hu@physi.uni-heidelberg.de
}

sensitivity of a Bragg-crystal spectrometer was used to obtain the polarization-dependent spectra. However, this technique cannot be applied for much heavier ions because high-energy $x$ rays emitted from such ions are out of the useful range of Bragg crystals. It is also impossible to directly observe the angular distribution with an EBIT because observation ports are usually opened only at $90^{\circ}$ with respect to the electron beam. On the other hand, direct angular distribution measurements for heavy ions have been done with an ion storage ring. For instance, Ma et al. [18] measured the angular distribution of $\mathrm{x}$ rays emitted in resonant transfer and excitation (RTE) in collisions of $\mathrm{H}$-like $\mathrm{U}$ and molecular hydrogen. RTE is a resonant recombination process similar to DR but the electron captured by an ion is not a free but a bound electron [19].

In our previous work [20], the magnetic-sublevel population of the autoionization state $\left[1 s 2 s^{2} 2 p_{1 / 2}\right]_{1}$ formed in DR of Li-like $\mathrm{Au}$ was experimentally determined with an EBIT in Tokyo [21]. As an alternative to direct angular distribution measurements, the alignment parameter that determines the angular distribution of the emitted $\mathrm{x}$ rays was obtained from the combination of the differential x-ray measurement at $90^{\circ}$ and the integral resonance-strength measurement through observing the ion-abundance ratios inside the EBIT. Comparison between the experimental result and the prediction by Fritzsche et al. clearly demonstrated the dominance of the Breit interaction in the electron-electron interaction in the high- $Z$ domain [20,22].

The Breit interaction effect is one of the quantum electrodynamics effects in electron-electron interaction, which includes magnetic interactions and retardation in the exchange of a single virtual photon between the electrons [23]. The Breit 
interaction is usually treated as a minor correction to the major term, i.e., the Coulomb interaction in atomic structure calculations [24,25]. On the other hand, in atomic collisions, the Breit interaction sometimes gives a prominent contribution. For instance, the resonance strengths of DR into highly charged heavy ions are greatly enhanced by the Breit interaction, as shown in our previous work [26] and also by Bernhardt et al. [27]. The Breit interaction also plays an important role in the quantum interference between resonant DR and nonresonant radiative recombination ( $R R)$ processes [28,29] as well as electron-impact excitation [30,31] and ionization [32,33].

In this paper, we present the experimental determination of the angular distribution for the same transition, i.e., $\left[1 s 2 s^{2} 2 p_{1 / 2}\right]_{1} \rightarrow\left[1 s^{2} 2 s^{2}\right]_{0}$, in $\operatorname{Pr}$ (atomic number $Z=59$ ) and Ho $(Z=67)$ to study the $Z$ dependence, in addition to the previous result for $\mathrm{Au}(Z=79)$. The angular distribution of the $\mathrm{x}$ rays emitted from DR of He-like ions is of intrinsically isotropic distribution and it has also been studied to examine the validity of the present experimental principle.

\section{EXPERIMENT}

The present experiments were performed with the EBIT at the University of Electro-Communications (UEC) in Tokyo [21]. The experimental method and procedure are similar to those used in our previous study [20], where the combination of the integral cross-section measurement through observing ionabundance ratios inside the trap $[29,34,35]$ and the differential cross section measurement through $\mathrm{x}$-ray observation at $90^{\circ}$ $[36,37]$ was used to obtain the angular distribution of the $x$ rays emitted in DR of Li-like $\mathrm{Au}(Z=79)$. In addition to the previous result, $\operatorname{Pr}(Z=59)$ and $\operatorname{Ho}(Z=67)$ were examined in the present study to investigate the $Z$ dependence. Pr and Ho were continuously injected into the EBIT with an effusion cell at $1100{ }^{\circ} \mathrm{C}$. In all of the measurements, the electron-beam current was $50 \mathrm{~mA}$ and the magnetic field used to compress the beam was $4 \mathrm{~T}$.

\section{A. Ion-abundance measurements}

The relative values of integral DR resonance strengths have been obtained by measuring the ion abundance in the EBIT as a function of electron-beam energy. The experimental method for the ion-abundance measurement is described in detail in our previous papers $[29,34,35]$. Briefly, the electron energy was stepwise scanned over the $K L_{12} L_{12}$ resonance [20] region by changing the potential at the cathode of the electron gun. The scan step was about $9 \mathrm{~V}$, and each step was kept for $10 \mathrm{~s}$ after the electron-beam energy was tuned. At each step, the ion counting was started after $2 \mathrm{~s}$ to ensure the equilibrium condition.

When the charge-state distribution inside the trap reaches equilibrium, the ion-abundance ratio between the adjacent ions is written as $[38,39]$

$$
n_{q-1} / n_{q}=\left[\sigma_{q}^{\mathrm{DR}}+\sigma_{q}^{\mathrm{RR}}+\left(e / j_{e}\right) n_{0} \sigma_{q}^{\mathrm{CX}} \bar{v}_{q}\right] / \sigma_{q-1}^{\mathrm{EI}},
$$

where $n_{q}$ is the number density of the ions inside the trap, $n_{0}$ the density of the neutrals, $j_{e}$ the density of the electron current, and $\bar{v}$ the mean velocity of the ions. $\sigma^{\mathrm{DR}}, \sigma^{\mathrm{RR}}, \sigma^{\mathrm{CX}}$, and $\sigma^{\mathrm{EI}}$ are the cross sections of DR, RR, single-electron exchange from neutral atoms, and electron-impact ionization, respectively.

Therefore, the relative DR cross section $\left[\sigma_{q}^{\mathrm{DR}}\right]=\sigma_{q}^{\mathrm{DR}} / \sigma_{q-1}^{\mathrm{EI}}$ can be obtained from the ion-abundance ratio $n_{q-1} / n_{q}$ between the ions with charge states of $(q-1)$ and $q$ using the following formula (valid only at equilibrium):

$$
\left.\left[\sigma_{q}^{\mathrm{DR}}\right]\right|_{E_{e}}=\left.\frac{n_{q-1}}{n_{q}}\right|_{E_{e}}-B\left(E_{e}\right),
$$

where $B\left(E_{e}\right)=\left[\sigma^{\mathrm{RR}}+\left(e / j_{e}\right) n_{0} \sigma_{q}^{\mathrm{CX}} \bar{v}_{q}\right] / \sigma_{q-1}^{\mathrm{EI}}$ denotes the smoothly varying background that contains the ionization, the nonresonant recombination, and the charge-exchange cross sections, and $E_{e}$ is the electron energy. DR is a resonant process, which takes place only at particular electron energies. The ion-abundance ratio changes dramatically at the resonance energies. However, nonsmooth structures may appear even at nonresonance energies, and they are considered as the effect of ion escape from the trap and multiple charge exchange. For the present experiments, the corrections for this effect will be described in detail in Sec. III.

The ion-abundance ratio was obtained by measuring the intensity of ions extracted from the EBIT in the leaky mode [26,34]. Although the ion abundance in the trap and that of the extracted ion can be different due to the different extraction efficiency depending on the charge state, we have confirmed that the ratio between adjacent charge states is consistent between the trapped and the extracted ions within 10\% [40]. However, this uncertainty does not affect the measurement of the magnetic-sublevel population because of the strength ratios between the different resonant states in the same charge state, so that the difference in the extraction efficiency is completely canceled.

\section{B. X-ray measurements}

X-ray observation has been commonly used to study DR processes with an electron-beam ion trap [14,41-47]. Our present method to obtain relative differential DR cross sections at $90^{\circ}$ is the same as that used in the previous study [48]. In brief, $\mathrm{x}$ rays emitted from the intermediate states produced by the DC were detected with a high-purity Ge detector placed at $90^{\circ}$ with respect to the electron-beam propagation direction while scanning the electron-beam energy over the $K L_{12} L_{12}$ DR resonance region. The energy scan was performed with a triangular time function applied at the ion-trap region with a scan frequency of $1 \mathrm{~Hz}$.

Figures 1(a) and 1(b) show two-dimensional plots of $\mathrm{x}$-ray spectra for Pr and Ho, respectively. The X-ray energy was calibrated using the radiation of radioisotopes, whereas the electron-beam energy was calibrated to the theoretical DR resonance energies. The diagonal bands in the figure correspond to $\mathrm{x}$ rays emitted by nonresonant $\mathrm{RR}$ into the $n=2$ shell. On the RR slice, several bright spots were observed, which correspond to $\mathrm{x}$ rays emitted by the $K L_{12} L_{12}$ DR. Each spot is assigned as indicated in Fig. 1.

\section{RESULTS AND DISCUSSION}

The results for He-like ions, normalized to the ratio $n_{\mathrm{Li}} / n_{\mathrm{He}}$ are shown in Fig. 2. Although there should be three dominant 

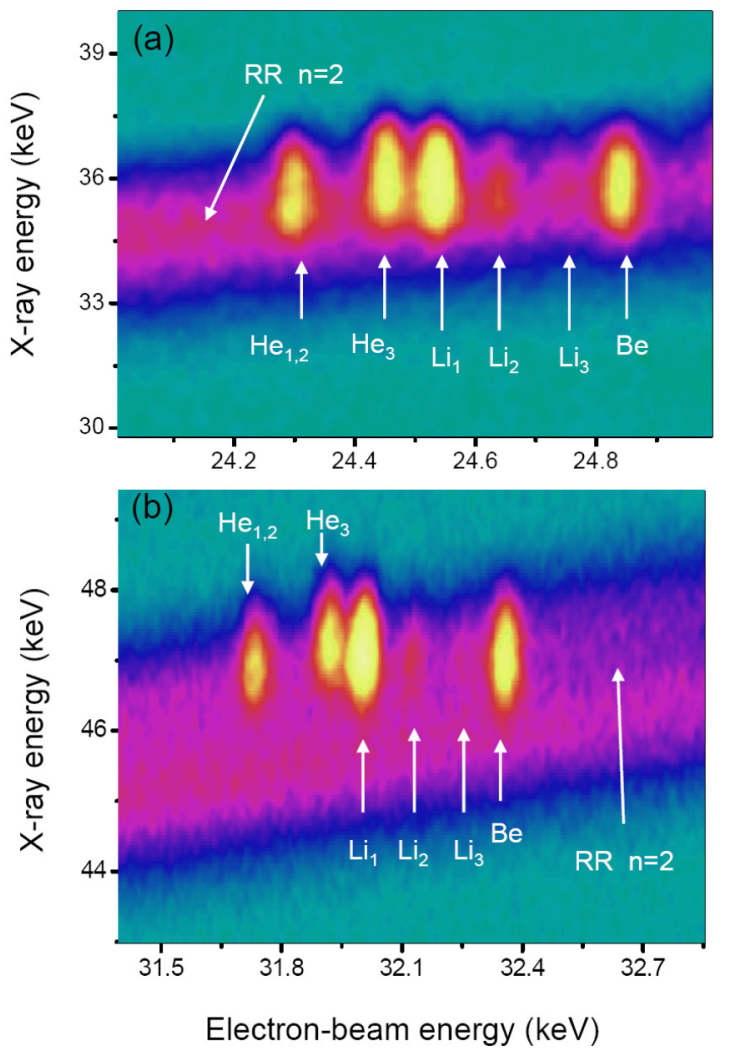

FIG. 1. (Color online) X-ray spectra from the $K L_{12} L_{12}$ DR of highly charged (a) Pr and (b) Ho ions. X-ray counts are plotted as the brightness of color as functions of electron-beam energy (horizontal axis) and x-ray energy (vertical axis). Bright spots correspond to $\mathrm{x}$-ray enhancement due to the DR. The labels represent the charge state responsible for each spot.

resonances in this region, only two prominent peaks are found due to insufficient electron-beam energy resolution. Thus, in the fitting procedure to obtain the relative resonance strength, the peak positions were fixed to the theoretical values calculated with the flexible atomic code (FAC) [49,50]. These theoretical values are listed in Table I, together with the theoretical resonance strengths with and without the Breit interaction. As seen in the table, the resonance strengths of the $\left[1 s 2 s^{2}\right]_{1 / 2}$ and the $\left[(1 s 2 s)_{0} 2 p_{1 / 2}\right]_{1 / 2}$ resonances are not too sensitive to the Breit interaction effect. However, the Breit interaction enhances the resonance strength of the $\left[(1 s 2 s)_{1} 2 p_{1 / 2}\right]_{1 / 2}$ resonance (the weak one) by almost two orders of magnitude while decreasing the resonance strength of the $\left[(1 s 2 s)_{1} 2 p_{1 / 2}\right]_{3 / 2}$ resonance. Due to its minor strength, the $\left[(1 s 2 s)_{1} 2 p_{1 / 2}\right]_{3 / 2}$ resonance was omitted in the fitting procedure.

Figure 3 shows $K$ x-ray intensities obtained by integrating the $2 \mathrm{D}$ plots shown in Fig. 1. Gaussian peaks fitted to the experimental data are also shown. Each peak corresponding to DR of He-like and Li-like ions is assigned with the same label as used in Figs. 2 and 4.

The integral resonance-strength ratios obtained from the ion-abundance measurements and the differential resonancestrength ratios obtained from the x-ray measurements are listed in Table II for He-like ions. Since all of the resonances
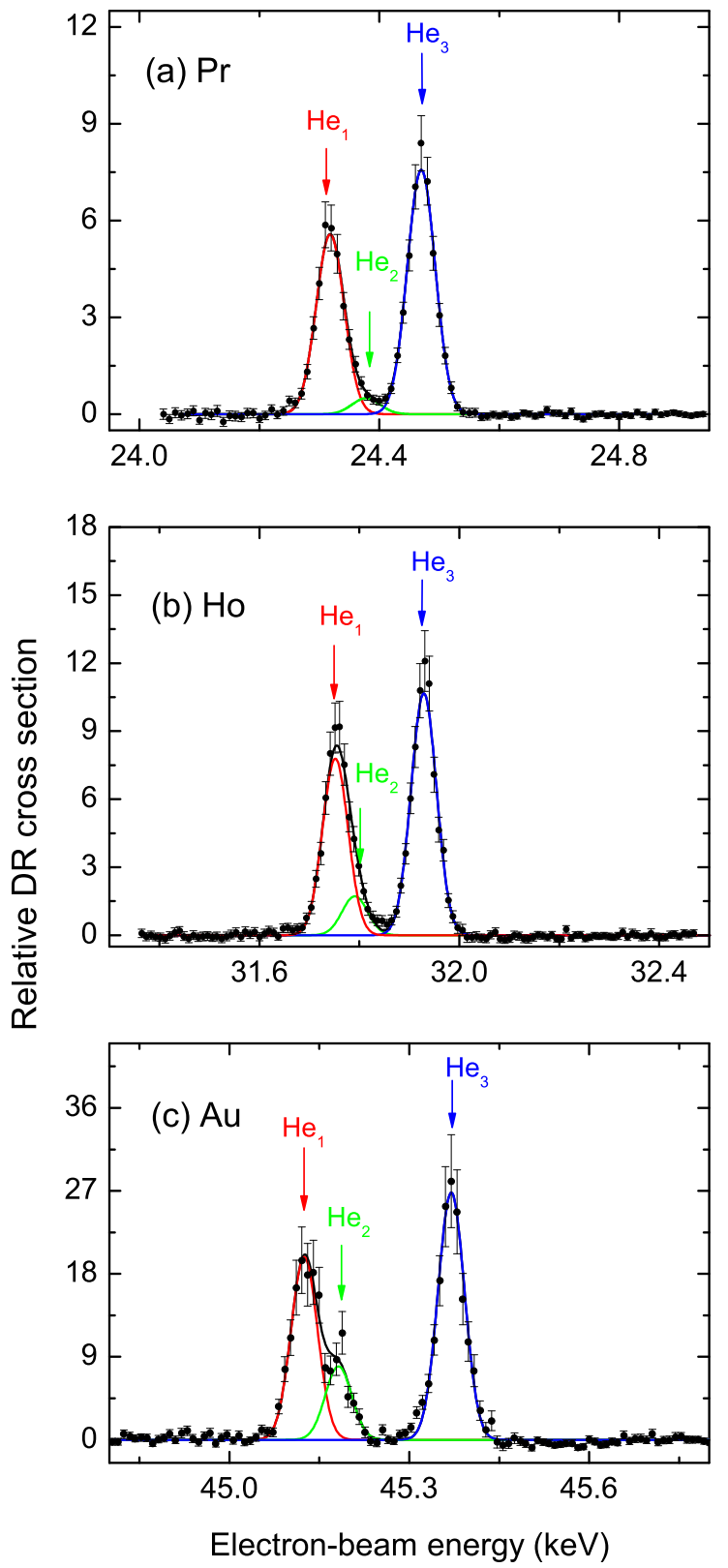

FIG. 2. (Color online) Relative DR cross section for He-like ions obtained from ion-abundance measurement for $\mathrm{Pr}(\mathrm{a}), \mathrm{Ho}(\mathrm{b})$, and $\mathrm{Au}$ (c). The Gaussian functions fitted to the experimental data are also shown by solid lines.

of interest have an intermediate state with a total angular momentum of $1 / 2$, the $x$-ray emission should have an isotropic distribution, i.e., the integral resonance-strength ratio and the differential resonance-strength ratio should be the same.

As shown in Table II, they are in agreement within experimental uncertainties, which implies that the present experimental method is valid. The experimental ratios also agree well with the theoretical ratios as shown in the table, and the theoretical ones were obtained from the calculated strengths with the Breit interaction which were taken from Table I.

Figure 4 shows the relative integral DR cross sections $\left\{\left[\sigma_{q}^{\mathrm{DR}}\right]\right.$ in Eq. (3) $\}$ of Li-like Pr and Ho, obtained from the 
TABLE I. Calculated $K L_{12} L_{12}$ DR resonance strengths of Helike ions. $E_{\text {res }}$ denotes the resonance energy in units of keV. $S^{\mathrm{C}+\mathrm{B}}$ and $S^{\mathrm{C}}$ denote the resonance strength with and without the Breit interaction, respectively, in units of $\mathrm{cm}^{2} \mathrm{eV}$.

\begin{tabular}{cccccc}
\hline \hline Ion & Label & Intermediate state & $E_{\text {res }}$ & $S^{\mathrm{C}+\mathrm{B}}$ & $S^{\mathrm{C}}$ \\
\hline $\mathrm{Pr}$ & $\mathrm{He}_{1}$ & {$\left[1 s 2 s^{2}\right]_{1 / 2}$} & 24.324 & $3.08 \times 10^{-20}$ & $2.64 \times 10^{-20}$ \\
& $\mathrm{He}_{2}$ & {$\left[(1 s 2 s)_{1} 2 p_{1 / 2}\right]_{1 / 2}$} & 24.361 & $0.30 \times 10^{-20}$ & $<1 \times 10^{-22}$ \\
& & {$\left[(1 s 2 s)_{1} 2 p_{1 / 2}\right]_{3 / 2}$} & 24.374 & $0.03 \times 10^{-20}$ & $0.51 \times 10^{-20}$ \\
& $\mathrm{He}_{3}$ & {$\left[(1 s 2 s)_{0} 2 p_{1 / 2}\right]_{1 / 2}$} & 24.476 & $4.94 \times 10^{-20}$ & $3.47 \times 10^{-20}$ \\
$\mathrm{Ho}$ & $\mathrm{He}_{1}$ & {$\left[1 s 2 s^{2}\right]_{1 / 2}$} & 31.745 & $3.17 \times 10^{-20}$ & $2.56 \times 10^{-20}$ \\
& $\mathrm{He}_{2}$ & {$\left[(1 s 2 s)_{1} 2 p_{1 / 2}\right]_{1 / 2}$} & 31.791 & $0.52 \times 10^{-20}$ & $0.01 \times 10^{-20}$ \\
& & {$\left[(1 s 2 s)_{1} 2 p_{1 / 2}\right]_{3 / 2}$} & 31.800 & $<1 \times 10^{-22}$ & $0.44 \times 10^{-20}$ \\
& $\mathrm{He}_{3}$ & {$\left[(1 s 2 s)_{0} 2 p_{1 / 2}\right]_{1 / 2}$} & 31.930 & $4.98 \times 10^{-20}$ & $3.37 \times 10^{-20}$ \\
$\mathrm{Au}$ & $\mathrm{He}_{1}$ & {$\left[1 s 2 s^{2}\right]_{1 / 2}$} & 45.101 & $3.35 \times 10^{-20}$ & $2.47 \times 10^{-20}$ \\
& & {$\left[(1 s 2 s)_{1} 2 p_{1 / 2}\right]_{3 / 2}$} & 45.157 & $0.05 \times 10^{-20}$ & $0.33 \times 10^{-20}$ \\
& $\mathrm{He}_{2}$ & {$\left[(1 s 2 s)_{1} 2 p_{1 / 2}\right]_{1 / 2}$} & 45.162 & $1.01 \times 10^{-20}$ & $0.04 \times 10^{-20}$ \\
& $\mathrm{He}_{3}$ & {$\left[(1 s 2 s)_{0} 2 p_{1 / 2}\right]_{1 / 2}$} & 45.349 & $5.32 \times 10^{-20}$ & $3.48 \times 10^{-20}$ \\
\hline \hline
\end{tabular}

ion-abundance ratio $n_{\mathrm{Be}} / n_{\mathrm{Li}}$. The electron-beam energy was calibrated with the theoretically calculated resonance energy. In each plot, the three peaks labeled $\mathrm{Li}_{1}$ to $\mathrm{Li}_{3}$ correspond to the $K L_{12} L_{12}$ DR of Li-like ions. The Gaussian functions fitted to the experimental data are also shown in the figure. The effects of ion escape and multiple charge exchange were
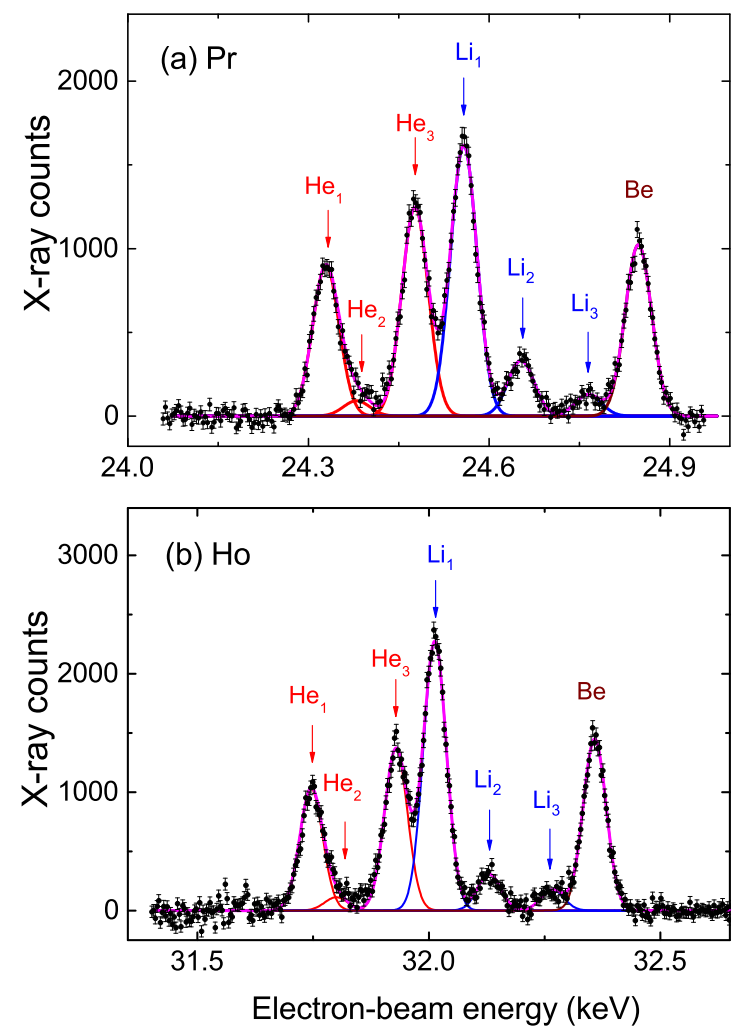

FIG. 3. (Color online) $K$ x-ray intensity plotted as a function of electron-beam energy in the $K L_{12} L_{12}$ resonance region for $\operatorname{Pr}$ (a) and Ho (b). The background corresponding to radiative recombination is subtracted. The labels represent the charge state responsible for the DR process that led to $\mathrm{x}$-ray enhancement.
TABLE II. $K L_{12} L_{12}$ DR resonance strength ratios of He-like ions. The error listed in the table corresponds to the error of the least-squares fitting weighted by the statistical uncertainties.

\begin{tabular}{ccrcc}
\hline \hline Ions & Ratios & Ion abundance & X ray & Calculated \\
\hline \multirow{2}{*}{ Pr } & $R_{\mathrm{He}_{1} / \mathrm{He}_{2}}$ & $11.17 \pm 1.44$ & $8.37 \pm 1.95$ & 10.27 \\
& $R_{\mathrm{He}_{1} / \mathrm{He}_{3}}$ & $0.74 \pm 0.19$ & $0.72 \pm 0.16$ & 0.62 \\
\multirow{2}{*}{$\mathrm{Ho}$} & $R_{\mathrm{He}_{1} / \mathrm{He}_{2}}$ & $4.53 \pm 1.75$ & $5.13 \pm 1.71$ & 6.10 \\
& $R_{\mathrm{He}_{1} / \mathrm{He}_{3}}$ & $0.73 \pm 0.16$ & $0.70 \pm 0.16$ & 0.64 \\
\multirow{2}{*}{$\mathrm{Au}$} & $R_{\mathrm{He}_{1} / \mathrm{He}_{2}}$ & $2.49 \pm 1.16$ & $4.13 \pm 1.32$ & 3.33 \\
& $R_{\mathrm{He}_{1} / \mathrm{He}_{3}}$ & $0.74 \pm 0.18$ & $0.73 \pm 0.17$ & 0.63 \\
\hline \hline
\end{tabular}

corrected for using the method as described in our previous paper [34]. For the correction, the $B\left(E_{e}\right)$ term in the Eq. (3) was modified as

$$
B^{*}\left(E_{e}\right)=\sum_{i=0}^{q_{\max }-q} \alpha_{i} n_{q+i} / n_{q},
$$

where $\alpha_{0}=\left[\sigma^{\mathrm{RR}}+\left(e / j_{e}\right)\left(n_{0} \sigma_{q}^{\mathrm{CX}} \bar{v}_{q}+\epsilon_{q}\right)\right] / \sigma_{q-1}^{\mathrm{EI}}$ and $\alpha_{i}=$ $\left(e / j_{e}\right)\left(n_{0} \sigma_{q+i}^{\mathrm{CX}(i+1)} \bar{v}_{q+i}+\epsilon_{q+i}\right) / \sigma_{q-1}^{\mathrm{EI}}$ for $i \geqslant 1, q_{\max }$ is the maximum charge state inside the trap, and $\epsilon$ is the ion escape rate. As mentioned in Ref. [34], the smoothly varying background function $B^{*}\left(E_{e}\right)$ can be distorted by the factor $n_{q+i} / n_{q}$ at energies which are not even at the DR resonances. In the present experiments, DR into He-like and Li-like ions was measured. For He-like ions, the background function becomes $B^{*}\left(E_{e}\right)_{\mathrm{He}}=\alpha_{0}$ and it is similar to the $B\left(E_{e}\right)$ in Eq. (3) apart from the term for ion escape. The background

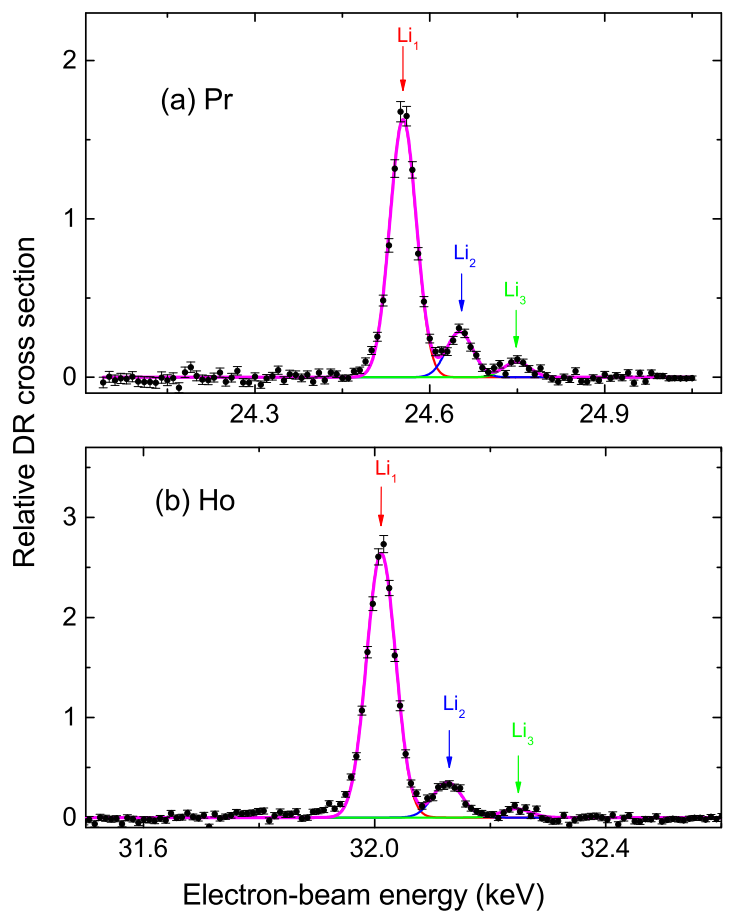

FIG. 4. (Color online) Relative DR cross section of Li-like ions obtained from the ion-abundance measurement for (a) Pr and (b) Ho. The Gaussian functions that were fitted to the experimental data are also shown by solid lines. 
TABLE III. $K L_{12} L_{12}$ DR resonance-strength ratios of Li-like ions. The error $(1 \sigma)$ listed in the table corresponds to the error of the leastsquares fitting weighted by the statistical uncertainties. $\mathrm{Li}_{1}$ and $\mathrm{Li}_{2}$ denote the resonances through the intermediate states of $\left[1 s 2 s^{2} 2 p_{1 / 2}\right]_{1}$ and $\left[1 s 2 s 2 p_{1 / 2}^{2}\right]_{1}$, respectively. $\mathrm{BR}_{\mathrm{Li}_{2}}$ are the branching ratios of the decay of the $\left[1 s 2 s 2 p_{1 / 2}^{2}\right]_{1}$ state to the two final states $\left[1 s^{2} 2 s 2 p_{1 / 2}\right]_{0} /\left[1 s^{2} 2 s 2 p_{1 / 2}\right]_{1}$. $W_{\mathrm{Li}_{1}}$ and $\mathcal{A}_{2}$ are the angular distribution function [Eq. (3)] and the alignment parameter [Eq. (4)].

\begin{tabular}{llllrr}
\hline \hline Ion & \multicolumn{1}{c}{$R_{\mathrm{Li}_{1} / \mathrm{Li}_{2}}^{\mathrm{x} \mathrm{ray}}$} & $R_{\mathrm{Li}_{1} / \mathrm{Li}_{2}}^{\text {ion }}$ & $\mathrm{BR}_{\mathrm{Li}_{2}}$ & $W_{\mathrm{Li}_{1}}$ & $\mathcal{A}_{2}$ \\
\hline $\mathrm{Pr}$ & $4.87 \pm 0.21$ & $5.66 \pm 0.26$ & $0.36 / 0.64$ & $0.86 \pm 0.05$ & $0.39 \pm 0.16$ \\
$\mathrm{Ho}$ & $7.50 \pm 0.39$ & $7.52 \pm 0.38$ & $0.35 / 0.65$ & $0.99 \pm 0.07$ & $0.01 \pm 0.20$ \\
$\mathrm{Au}$ & $13.0 \pm 0.9$ & $12.3 \pm 0.9$ & $0.34 / 0.66$ & $1.06 \pm 0.11$ & $-0.17 \pm 0.31$ \\
\hline \hline
\end{tabular}

function $B^{*}\left(E_{e}\right)_{\mathrm{He}}$ is independent of the factor $n_{q+i} / n_{q}$, and as a result, there are no additional structures in the DR resonances. This was confirmed by the experimental data. For Li-like ions, $i$ can be 0 or 1 , and the background function is $B^{*}\left(E_{e}\right)_{\mathrm{Li}}=\alpha_{0}+\alpha_{1} n_{\mathrm{He}} / n_{\mathrm{Li}}$. The $B^{*}\left(E_{e}\right)_{\mathrm{Li}}$ functions of DR into Li-like $\operatorname{Pr}$ and Ho had additional structures due to the great changes of the $n_{\mathrm{He}} / n_{\mathrm{Li}}$ factor which take place at the resonance energies of DR into He-like ions. There are no additional structures for DR into Li-like $\mathrm{Au}$. This can be understood as follows: the relatively low abundance of He-like Au gave a small value for the $n_{\mathrm{He}} / n_{\mathrm{Li}}$ factor, and as a result, the $n_{\mathrm{He}} / n_{\mathrm{Li}}$ factor could not cause any additional structures in the background function. $\alpha_{i}$ was obtained by solving Eq. (4) in Ref. [34] in the off-resonance region, and then the obtained $\alpha_{i}$ was used to remove the additional structures even though they were superposed on the DR resonances.

The resonance-strength ratios between the $\mathrm{Li}_{1}$ and $\mathrm{Li}_{2}$ resonances are listed in Table III both for the integral resonance strength obtained from the ion-abundance measurements $\left(R_{\mathrm{Li}_{1} / \mathrm{Li}_{2}}^{\mathrm{ion}}\right)$ and for the differential resonance strength obtained from the x-ray measurements $\left(R_{\mathrm{Li}_{1} / \mathrm{Li}_{2}}^{\mathrm{x} \text { ray }}\right)$. The tabulated errors of the integral resonance-strength ratios between the $\mathrm{Li}_{1}$ and $\mathrm{Li}_{2}$ resonances include the systematic uncertainties associated with the correction procedure used in the cases of Pr and Ho. The angular distribution factor $W(\theta)$ at $\theta=90^{\circ}(\theta$ denotes the $\mathrm{x}$-ray emission angle with respect to the electron-beam propagation direction) for the $\mathrm{x}$-ray emission in the $\mathrm{Li}_{1}$ resonance can be obtained as we did before for $\mathrm{Au}$ from the following equation [20]:

$$
W_{\mathrm{Li}_{1}}\left(90^{\circ}\right)=R_{\mathrm{Li}_{1} / \mathrm{Li}_{2}}^{\mathrm{x} \text { ray }} / R_{\mathrm{Li}_{1} / \mathrm{Li}_{2}}^{\text {ion }} .
$$

This equation is valid under the condition that the branching ratio for the decay of the intermediate state $\left[1 s 2 s 2 p_{1 / 2}^{2}\right]_{1}$ in the $\mathrm{Li}_{2}$ resonance to the final states $\left[1 s^{2} 2 s 2 p_{1 / 2}\right]_{0}$ and $\left[1 s^{2} 2 s 2 p_{1 / 2}\right]_{1}$ should be $1 / 3$ and $2 / 3$, respectively. We confirmed through the FAC calculation that this condition holds not only for Au but also for Pr and Ho as shown in Table III. The alignment parameter $\mathcal{A}_{2}$ of the intermediate autoionization state can be obtained from the angular distribution factor $W\left(90^{\circ}\right)$ as

$$
\mathcal{A}_{2}=2 \sqrt{2}\left[1-W\left(90^{\circ}\right)\right] \text {. }
$$

The present experimental results of the alignment parameter are plotted in Fig. 5 together with the theoretical prediction by Fritzsche et al. [22]. The agreement between the present experiment and theory demonstrates the strong $Z$-dependent tendency due to the Breit interaction. The alignment parame- ters were also calculated using the FAC, and they reproduced the prediction by Fritzsche et al. quite well.

$\mathcal{A}_{2}$ is defined by the magnetic-sublevel population of the intermediate state, and for $J=1$ intermediate states, it is expressed as

$$
\mathcal{A}_{2}=\sqrt{2} \frac{\sigma_{ \pm 1}-\sigma_{0}}{2 \sigma_{ \pm 1}+\sigma_{0}}
$$

where $\sigma_{M_{J}}$ denotes the population of the magnetic sublevel with the magnetic quantum number $M_{J}$. The magneticsublevel population is proportional to the DC resonance strength for the individual magnetic sublevels. Figure 6 shows the DC resonance strengths $\Omega_{0}$ and $\Omega_{ \pm 1}$ for the magnetic sublevels $M_{J}=0$ and $M_{J}= \pm 1$, respectively. When only the Coulomb interaction is considered, the DC resonance strengths for both $M_{J}= \pm 1$ and $M_{J}=0$ sublevels have an insignificant $Z$ dependence. Especially at around $Z=53, \Omega_{0}$ is negligibly small compared to $\Omega_{ \pm 1}$, which results in $\mathcal{A}_{2} \approx \sqrt{2} / 2 \approx 0.71$ as indicated by the upper dashed line in Fig. 5. This can be understood in the nonrelativistic limit. In this limit, only the magnetic sublevels with $M_{J}= \pm 1$ can be populated in dielectronic recombination [22]. Although $\Omega_{0}$ increases with $Z$, the dependence on $Z$ is gradual; thus the $Z$ dependence of

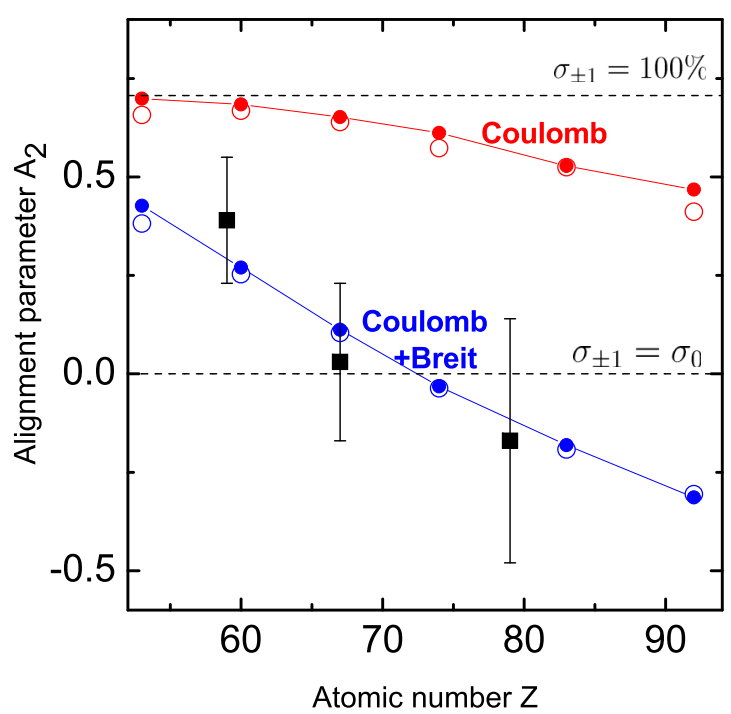

FIG. 5. (Color online) Alignment paramenters $\mathcal{A}_{2}$ for the intermediate state $\left[1 s 2 s^{2} 2 p_{1 / 2}\right]_{1}$ in DR of Li-like Pr, Ho, and Au. The experimental results are shown by black squares. Theoretical values are plotted as red and blue dots. The present calculation by the FAC is also plotted with the red and blue circles. 


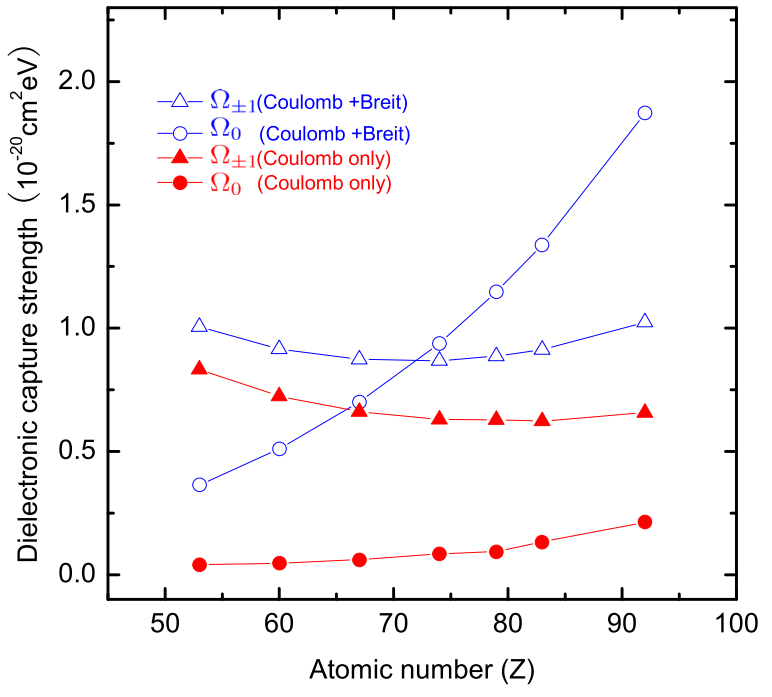

FIG. 6. (Color online) Calculated dielectronic capture strengths for each magnetic sublevel.

$\mathcal{A}_{2}$ is also gradual as shown in Fig. 5 when only the Coulomb interaction is considered.

On the other hand, when the Breit interaction is included, both $\Omega_{0}$ and $\Omega_{ \pm 1}$ are modified as shown in Fig. 6 . In particular, the modification to $\Omega_{0}$ is substantial in both the absolute value and the $Z$-dependent tendency. It has a much smaller value compared with $\Omega_{ \pm 1}$ even at around $Z=53$, and the strong $Z$ dependence makes it larger than $\Omega_{ \pm 1}$ for higher $Z$. The reversal of the magnitude relationship between $\Omega_{0}$ and $\Omega_{ \pm 1}$ results in a change of the sign of $\mathcal{A}_{2}$, i.e., the change of the angular distribution from a function with a minimum at $90^{\circ}$ to one with a maximum at $90^{\circ}$. Thus, the substantial modification to $\Omega_{0}$ is responsible for the strong $Z$-dependent tendency of the alignment parameter $\mathcal{A}_{2}$.

It should be noted that the external magnetic and electric fields applied can modify the resonance strength and the magnetic-sublevel distribution. Indeed several previous theoretical and experimental studies demonstrated that the DR resonance strength can be affected by external fields [51-54]. However, those studies are limited to DR processes into high Rydberg states of relatively light ions, which have relatively large sensitivity for external fields. On the other hand, only $K$ and $L$-shell electrons, which are strongly bound in the nuclear field, are involved in this study. The external field applied to the trap region is many orders of magnitude weaker than the nuclear field, so that it should have little effect on either the resonance strength or the sublevel population distribution. In addition, since the present external magnetic field has the same direction as the electron beam, it should not redistribute the magnetic-sublevel population even if it is strong.

\section{CONCLUSIONS}

We have observed the angular distribution of the $\left[1 s 2 s^{2} 2 p_{1 / 2}\right]_{1} \rightarrow\left[1 s^{2} 2 s^{2}\right]_{0}$ transitions in dieletronic recombination for Li-like Pr, Ho, and Au with the Tokyo electron-beam ion trap. The alignment parameters of the autoionization states $\left[1 s 2 s^{2} 2 p_{1 / 2}\right]_{1}$ were obtained and compared with the recent theoretical prediction [22]. The present experimental result is not only a supplement to our previous work [20], but also demonstrates a strong $Z$-dependent tendency due to the Breit interaction effect on the angular distribution of $\mathrm{x}$-ray emissions, i.e., the magnetic-sublevel population, in dielectronic recombination.

\section{ACKNOWLEDGMENTS}

This work was supported by JSPS KAKENHI Grant No. 21340111. Z.H. would like to express acknowledgment for the support by the Foundation of Science and Technology on Plasma Physics Laboratory (Grant No. 9140C6804010906) and the Science and Technology Foundation of the China Academy of Engineering Physics (Grant No. 2013A0102002).
[1] U. Fano and J. H. Macek, Rev. Mod. Phys. 45, 553 (1973).

[2] J. C. Kieffer, J. P. Matte, H. Pépin, M. Chaker, Y. Beaudoin, T. W. Johnston, C. Y. Chien, S. Coe, G. Mourou, and J. Dubau, Phys. Rev. Lett. 68, 480 (1992).

[3] J. Dubau, M. K. Inal, and A. M. Urnov, Phys. Scr., T 65, 179 (1996).

[4] H. Yoneda, N. Hasegawa, S.-I. Kawana, and K.-I. Ueda, Phys. Rev. E 56, 988 (1997).

[5] Y. Inubushi, T. Kai, T. Nakamura, S. Fujioka, H. Nishimura, and K. Mima, Phys. Rev. E 75, 026401 (2007).

[6] K. Widmann, P. Beiersdorfer, V. Decaux, S. R. Elliott, D. Knapp, A. Osterheld, M. Bitter, and A. Smith, Rev. Sci. Instrum. 66, 761 (1995).

[7] M. Bitter, M. F. Gu, L. A. Vainshtein, P. Beiersdorfer, G. Bertschinger, O. Marchuk, R. Bell, B. LeBlanc, K. W. Hill, D. Johnson, and L. Roquemore, Phys. Rev. Lett. 91, 265001 (2003).
[8] M. K. Inal and J. Dubau, J. Phys. B 22, 3329 (1989).

[9] M. H. Chen and J. H. Scofield, Phys. Rev. A 52, 2057 (1995).

[10] S. Zakowicz, W. Scheid, and N. Grün, Nucl. Instrum. Methods Phys. Res., Sect. B 205, 386 (2003).

[11] S. Fritzsche, N. M. Kabachnik, and A. Surzhykov, Phys. Rev. A 78, 032703 (2008).

[12] S. Fritzsche, A. Surzhykov, and T. Stöhlker, Phys. Scr., T 144, 014002 (2011).

[13] D. A. Knapp, R. E. Marrs, M. A. Levine, C. L. Bennett, M. H. Chen, J. R. Henderson, M. B. Schneider, and J. H. Scofield, Phys. Rev. Lett. 62, 2104 (1989).

[14] A. J. Smith, P. Beiersdorfer, K. Widmann, M. H. Chen, and J. H. Schofield, Phys. Rev. A 62, 052717 (2000).

[15] D. R. DeWitt, R. Schuch, H. Gao, W. Zong, S. Asp, C. Biedermann, M. H. Chen, and N. R. Badnell, Phys. Rev. A 53, 2327 (1996).

[16] G. Kilgus, J. Berger, P. Blatt, M. Grieser, D. Habs, B. Hochadel, E. Jaeschke, D. Krämer, R. Neumann, G. Neureither, W. Ott, 
D. Schwalm, M. Steck, R. Stokstad, E. Szmola, A. Wolf, R. Schuch, A. Müller, and M. Wagner, Phys. Rev. Lett. 64, 737 (1990).

[17] A. S. Shlyaptseva, R. C. Mancini, P. Neill, P. Beiersdorfer, J. R. Crespo López-Urrutia, and K. Widmann, Phys. Rev. A 57, 888 (1998).

[18] X. Ma, P. H. Mokler, F. Bosch, A. Gumberidze, C. Kozhuharov, D. Liesen, D. Sierpowski, Z. Stachura, T. Stöhlker, and A. Warczak, Phys. Rev. A 68, 042712 (2003).

[19] S. Zakowicz, Z. Harman, N. Grün, and W. Scheid, Phys. Rev. A 68, 042711 (2003).

[20] Z. Hu, X. Han, Y. Li, D. Kato, X. Tong, and N. Nakamura, Phys. Rev. Lett. 108, 073002 (2012).

[21] N. Nakamura, J. Asada, F. J. Currell, T. Fukami, T. Hirayama, K. Motohashi, T. Nagata, E. Nojikawa, S. Ohtani, K. Okazaki, M. Sakurai, H. Shiraishi, S. Tsurubuchi, and H. Watanabe, Phys. Scr., T 73, 362 (1997).

[22] S. Fritzsche, A. Surzhykov, and T. Stöhlker, Phys. Rev. Lett. 103, 113001 (2009).

[23] G. Breit, Phys. Rev. 34, 553 (1929).

[24] J. B. Mann and W. R. Johnson, Phys. Rev. A 4, 41 (1971).

[25] V. V. Karasiev, E. V. Ludeña, and O. A. Shukruto, Phys. Rev. A 69, 052509 (2004).

[26] N. Nakamura, A. P. Kavanagh, H. Watanabe, H. A. Sakaue, Y. Li, D. Kato, F. J. Currell, and S. Ohtani, Phys. Rev. Lett. 100, 073203 (2008).

[27] D. Bernhardt, C. Brandau, Z. Harman, C. Kozhuharov, A. Müller, W. Scheid, S. Schippers, E. W. Schmidt, D. Yu, A. N. Artemyev, I. I. Tupitsyn, S. Böhm, F. Bosch, F. J. Currell, B. Franzke, A. Gumberidze, J. Jacobi, P. H. Mokler, F. Nolden, U. Spillman, Z. Stachura, M. Steck, and T. Stöhlker, Phys. Rev. A 83, 020701 (2011).

[28] X. M. Tong, N. Nakamura, S. Ohtani, T. Watanabe, and N. Toshima, Phys. Rev. A 80, 042502 (2009).

[29] N. Nakamura, A. P. Kavanagh, H. Watanabe, H. A. Sakaue, Y. Li, D. Kato, F. J. Currell, X.-M. Tong, T. Watanabe, and S. Ohtani, Phys. Rev. A 80, 014503 (2009).

[30] C. J. Fontes, D. H. Sampson, and H. L. Zhang, Phys. Rev. A 47, 1009 (1993).

[31] A. Gumberidze, D. B. Thorn, C. J. Fontes, B. Najjari, H. L. Zhang, A. Surzhykov, A. Voitkiv, S. Fritzsche, D. Banaś, H. Beyer, W. Chen, R. D. DuBois, S. Geyer, R. E. Grisenti, S. Hagmann, M. Hegewald, S. Hess, C. Kozhuharov, R. Märtin, I. Orban, N. Petridis, R. Reuschl, A. Simon, U. Spillmann, M. Trassinelli, S. Trotsenko, G. Weber, D. F. A. Winters, N. Winters, D. Yu, and T. Stöhlker, Phys. Rev. Lett. 110, 213201 (2013).

[32] R. E. Marrs, S. R. Elliott, and D. A. Knapp, Phys. Rev. Lett. 72, 4082 (1994).
[33] C. J. Fontes, D. H. Sampson, and H. L. Zhang, Phys. Rev. A 59, 1329 (1999).

[34] H. Watanabe, H. Tobiyama, A. P. Kavanagh, Y. M. Li, N. Nakamura, H. A. Sakaue, F. J. Currell, and S. Ohtani, Phys. Rev. A 75, 012702 (2007).

[35] A. P. Kavanagh, H. Watanabe, Y. M. Li, B. E. O'Rourke, H. Tobiyama, N. Nakamura, S. McMahon, C. Yamada, S. Ohtani, and F. J. Currell, Phys. Rev. A 81, 022712 (2010).

[36] H. Watanabe, F. J. Currell, H. Kuramoto, Y. M. Li, S. Ohtani, B. O'Rourke, and X. M. Tong, J. Phys. B 34, 5095 (2001).

[37] H. Tobiyama, H. Nohara, A. P. Kavanagh, N. Nakamura, H. Watanabe, H. Sakaue, Y. M. Li, F. J. Currell, and S. Yamada, C Ohtani, J. Phys.: Conf. Ser. 58, 239 (2007).

[38] B. M. Penetrante, J. N. Bardsley, D. DeWitt, M. Clark, and D. Schneider, Phys. Rev. A 43, 4861 (1991).

[39] R. Ali, C. P. Bhalla, C. L. Cocke, M. Schulz, and M. Stockli, Phys. Rev. A 44, 223 (1991).

[40] Z. Hu, Y. Ishiguro, H. Watanabe, S. Ohtani, and N. Nakamura, Nucl. Instrum. Methods Phys. Res., Sect. B 298, 96 (2013).

[41] D. A. Knapp, P. Beiersdorfer, M. H. Chen, J. H. Scofield, and D. Schneider, Phys. Rev. Lett. 74, 54 (1995).

[42] Y. Zou, J. R. Crespo López-Urrutia, and J. Ullrich, Phys. Rev. A 67, 042703 (2003).

[43] K. Yao, Z. Geng, J. Xiao, Y. Yang, C. Chen, Y. Fu, D. Lu, R. Hutton, and Y. Zou, Phys. Rev. A 81, 022714 (2010).

[44] Z. Hu, Y. Li, A. Yamazaki, and N. Nakamura, Phys. Scr., T 144, 014047 (2011).

[45] C. Beilmann, P. H. Mokler, S. Bernitt, C. H. Keitel, J. Ullrich, J. R. Crespo López-Urrutia, and Z. Harman, Phys. Rev. Lett. 107, 143201 (2011).

[46] G. Xiong, J. Zhang, Z. Hu, N. Nakamura, Y. Li, X. Han, J. Yang, and B. Zhang, Phys. Rev. A 88, 042704 (2013).

[47] C. Beilmann, Z. Harman, P. H. Mokler, S. Bernitt, C. H. Keitel, J. Ullrich, and J. R. Crespo López-Urrutia, Phys. Rev. A 88 , 062706 (2013).

[48] Z. Hu, Y. Li, and N. Nakamura, Phys. Rev. A 87, 052706 (2013).

[49] M. F. Gu, Astrophys. J. 582, 1241 (2003).

[50] M. F. Gu, Can. J. Phys. 86, 675 (2008).

[51] F. Robicheaux and M. S. Pindzola, Phys. Rev. Lett. 79, 2237 (1997).

[52] F. Robicheaux, M. S. Pindzola, and D. C. Griffin, Phys. Rev. Lett. 80, 1402 (1998).

[53] D. C. Griffin, F. Robicheaux, and M. S. Pindzola, Phys. Rev. A 57, 2708 (1998).

[54] T. Bartsch, S. Schippers, A. Müller, C. Brandau, G. Gwinner, A. A. Saghiri, M. Beutelspacher, M. Grieser, D. Schwalm, A. Wolf, H. Danared, and G. H. Dunn, Phys. Rev. Lett. 82, 3779 (1999). 